\title{
Possibilities and limitations of the organization of anticorrosion protection at gas production facilities
}

\author{
Dmitry Zapevalov ${ }^{1}$, Ruslan Vagapov ${ }^{1}$ \\ ${ }^{1}$ LLC «Gazprom VNIIGAZ», Corporative scientific-technical center of corrosion monitoring and corrosion protection, 142717, Moscow \\ region, Russian Federation
}

\begin{abstract}
Products of several going and promising onshore and offshore hydrocarbon extraction sites both in Russia and foreign countries are known due to elevated level of corrosive $\mathrm{CO}_{2}$. The article analyzes present procedures aimed at rating corrosive hazard of extracted fluids, selection and implementation of measures for protection of equipment and pipelines against of inner corrosion. Onshore and offshore facilities work within specific environment, which affects arrangement of corrosion protection and corrosion monitoring systems. Presence of $\mathrm{CO}_{2}$ in the extracted products together with other factors stimulate intensive growth of corrosion, and require attentive and reasonable attitude to choose engineering solutions providing safe and reliable operation of production facilities.
\end{abstract}

\section{Introduction}

Reliable and safe operation of production facilities, while ensuring the required service life of equipment and pipelines, is a systematic task in the operation of hydrocarbon fields. Currently, one of the key areas of development of the Russian resource base for gas and gas condensate is the development of fields characterized by an increased $\mathrm{CO}_{2}$ content [1]. Assessment and forecast of the influence of this corrosion factor significantly affect the choice of design solutions and the features of field operation in conditions of carbon dioxide corrosion.

Under such conditions one of the technically justified and efficient methods of protection is use of inhibitors that adsorb as protective films at a metal to prevent its corrosion. At the same time inhibitor protection seems to be one of the most appropriate and cost-efficient ways to address this problem [2].

The purpose of the work is to analyze the corrosive operating conditions and applied inhibitory corrosion protection to develop and optimize solutions for anticorrosion protection and organization of corrosion monitoring at gas production facilities.

\section{Corrosion activity of medium}

In the initial assessment of the corrosiveness of media during carbon dioxide corrosion, partial pressures $\mathrm{P}_{\mathrm{CO} 2}$, mineralization, $\mathrm{pH}$ and other parameters are important. The final assessment is carried out based on the results of simulation tests (laboratory and autoclave) in corrosive conditions close to real ones, with the determination of the corrosion rate of the medium.
According to the requirements of regulatory documents for onshore and offshore facilities, a medium with a corrosion rate higher than $0.1 \mathrm{~mm} /$ year refers to systems with high corrosivity, which requires the use of corrosion protection, for example, corrosion inhibitors [3-4].

The analysis of data on the current content of $\mathrm{CO}_{2}$ in the produced products is carried out with the determination of the partial pressure of $\mathrm{CO}_{2}$. Additionally, in the presence of forecast data and plans for the further development of the production facility, the potential change (increase / decrease) in the $\mathrm{CO}_{2}$ content over time is estimated. This is important for the correct selection of corrosion protection measures, taking into account the operating conditions throughout the life cycle of mining equipment and pipelines.

The determination of pressure and temperature is carried out, which can also undergo changes during the operation of gas production facilities. A decrease in the total pressure, which affects the change in the partial pressure of $\mathrm{CO}_{2}$, can occur both during time of operation and during movement through the pipeline system.

Another important aspect in the selection of corrosion test media is the mineral composition and $\mathrm{pH}$ of the aqueous medium. Some of the starting position of the data processing and analysis methods in the selection of the aqueous phase to the gas field have been previously outlined [5]. For an operating field, there is usually a large set of data on analysis of aquatic environments, but for projected production facilities, on the contrary, a lack of data. The water phase can be either condensation or reservoir in nature. An example of the analysis and processing of the data set on the mineralization of the aqueous phase is shown in figure 1.

* Corresponding author: R_Vagapov@vniigaz.gazprom.ru 
All of the above parameters are the main factors affecting corrosion. They are also used as criteria in assessing the corrosivity of the operating environment.

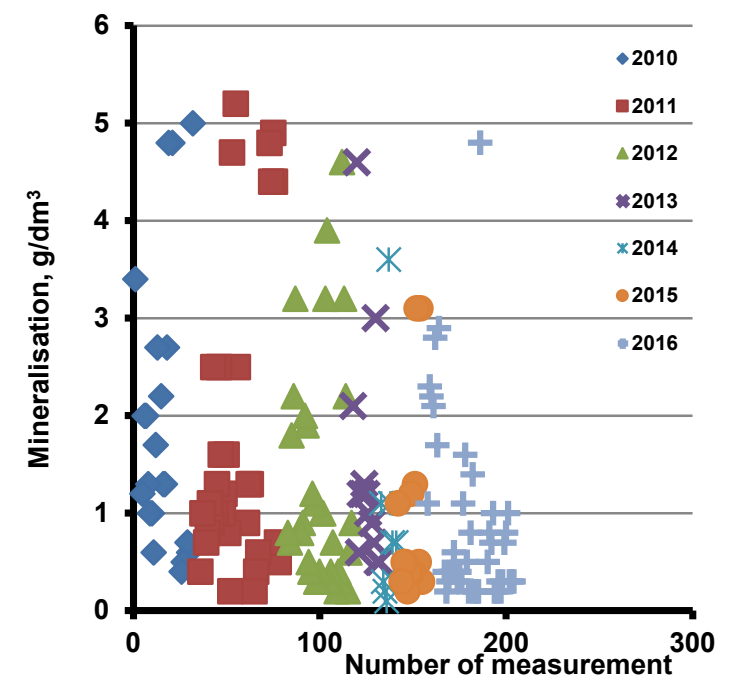

Fig. 1. The results of studies of water samples by mineralization [5].

During the operation of the mining object, an increase in the corrosion rate may occur. The reasons for the intensification of corrosion processes can be different circumstances, and they can be both temporary and permanent. For example, the ingress of process fluids (drilling fluid, etc.) can lead to a short-term increase in the salinity of the aqueous medium, and the acid solution will lead to a decrease in the $\mathrm{pH}$-factor of the aqueous medium. In figure 2 shows the data of corrosion tests of drilling fluids and aqueous electrolytes, which are used for its preparation. It can be seen that they can be corrosive: the corrosion rate is in the range from 1 to 2.8 $\mathrm{mm} /$ year. However, the increase in salinity can be constant, and then this will indicate a change in the nature of the water phase and its transition from condensation to formation. Therefore, the dynamics of changes in corrosion parameters in time is estimated in order to know whether they are constant or temporary.

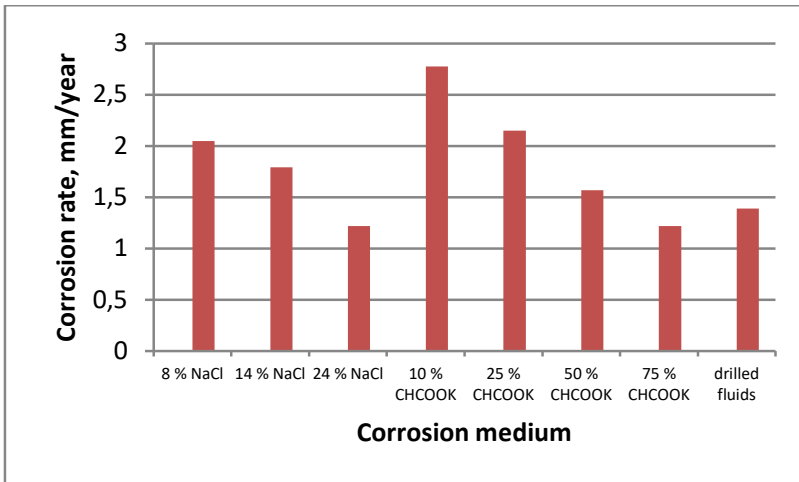

Fig. 2 Corrosion rate of the low carbon steel in drilling fluids and in the electrolyte, which can be used for its preparation.

In a gas pipeline, corrosion may occur [6]:

- in the lower part of the pipe when moisture accumulates (bottom-of-line corrosion, BOL).
- in the upper part of the pipe, moisture condensation (top-of-line corrosion, TOL).

- in places of moisture accumulation (cracks, gaps, stagnant zones).

The most intense corrosive effect is in the form of TOL corrosion, which is characterized by the localization of the corrosion process with the formation of pits and other local damages. It is corrosion processes that occur under conditions of moisture condensation that are an operational feature and are characteristic only of gas facilities, and distinguish it from oil fields in terms of the development of corrosion. For the conditions for the start and flow of the process to arise, it is necessary that the produced formation gas has a certain moisture content, and at the same time, thermobaric conditions would be created for the separation of water from the gaseous medium, with its condensation on the inner surface of the upper component of the pipe [7-9].

\section{Corrosion inhibitors}

The long-term operational practice of using inhibitors for corrosion protection confirms the reliability and effectiveness of this method of protection in various conditions.

However, there are no inhibitors that are sufficiently effective in a wide range of operating conditions. This explains the need for the selection of inhibitors for each specific object and operating conditions. The choice of inhibitors and inhibition methods for specific corrosion conditions is a very difficult task. It is advisable to carry out a preliminary selection of the inhibitor in environments similar to the field one: in laboratory conditions, in autoclave conditions, and only then in the conditions of the operating facility [1].

In order to effectively protect equipment, the inhibitor should be evenly delivered to its entire surface in contact with aggressive media. The main supply agent of continuous inhibition of gas production facilities is the medium itself. This implies that the inhibitor must be soluble or well dispersed in it. Therefore, an important technological property of inhibitors is the ability to dissolve and protect in various process fluids. As noted earlier, the main media are hydrocarbon and aqueous (water-glycolic) solution, in which corrosion inhibitors should be tested.

In the previous report [6] we discuss that the operating conditions of gas and oil fields are vary, which also effects on the requirements to corrosion inhibitors.

The inhibitors applied during the operation of gas equipment and pipelines should satisfy a number of protecting and technological requirements [6]:

- should be soluble or dispersible at least in one of solvents (water or brine; alcohol; hydrocarbons);

- tshould not create emulsions in mixture of water and condensates;

- should ensure a highly protective effect;

- should prevent the formation of pitting;

- should be stable to low temperature;

- should be non-toxic; 
- have a strong after-effects.

Inhibitors for oil fields cannot be used in a gas fields without modification: the active basis of the corrosion protection reagent may remain the same, but additives are needed that determine the inhibitor's technological parameters: solubility, freezing point, tendency to form an emulsion, and others [6].

Inhibitors used in a gas production, along with the high protective properties must have certain technological properties (solubility/dispersibility, freezing point, characterized by the absence of a negative effect on the separation of water-hydrocarbon emulsion) $[5,10]$.

Examples of precipitation in solutions of corrosion inhibitors in different solvents are shown on figure 3, which limits the use of solutions of such inhibitors.
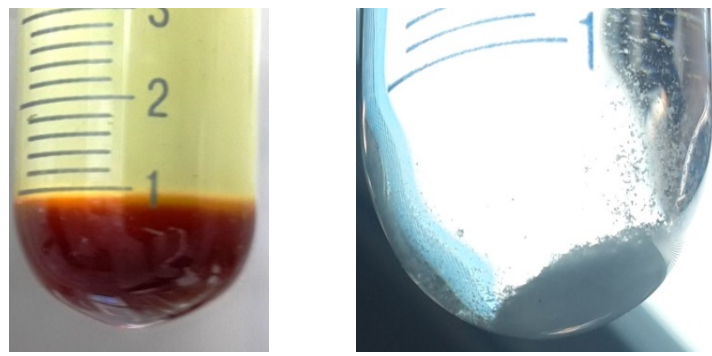

Fig. 3. Precipitation in a solution of a corrosion inhibitor in different solvents.

\section{Corrosion monitoring}

Corrosion monitoring methods are divided into direct and indirect methods [11]:

Direct methods include:

- exposure of gravimetric coupons.

- ER-probes (the use of LPR-probes is limited due to the lack of an electrically conductive medium (aqueous electrolyte));

- diagnostic methods (In-line-inspection, Wall Thickness, Ultrasonic Testing, Radiography Testing).

- conducting tests of varying degrees of approximation to real field conditions (laboratory, autoclave, operational).

Indirect methods include:

- determination of the content of aggressive components of gas and liquid media (partial pressures, ion concentrations, mechanical impurities, etc.).

- determination of changes in the content of corrosion products and corrosion inhibitor in operating environments.

- determination of technological parameters (temperature, flow rate, flow regime, etc.).

To obtain a reliable assessment based on the results of corrosion monitoring, the data obtained by different methods of corrosion monitoring should be correctly interpreted, and also correctly correlated and compared with each other [12]. For this, it is necessary to determine the criteria by which the assessment of the corrosiveness of the medium and the effectiveness of inhibitor protection will be carried out. These criteria are the values of corrosion rate, thinning (pipe wall thickness), the presence and depth of defects, the content of corrosion products and corrosion inhibitor in the liquid, the content of corrosive gas in the produced hydrocarbons, etc. [13].

It should be noted that one of the main diagnostic methods is in-line-inspection, but it is rarely performed [14]. In such conditions, to control pipelines, a rational option is to obtain data using the entire range of possible monitoring methods. This will make it possible to assess the technical condition of objects and form empirical models on their basis. Additional information sources include conducting simulation tests that simulate operating conditions in pipeline sections inaccessible to in-line-inspection.

The main tool for thickness measurement is the ultrasonic testing. In conditions of localized corrosion, ultrasonic testing of wall thickness is used in conjunction with radiography testing to control the size and depth and change them over time. If for cases of hydrogen sulfide corrosion, when both general and local corrosion can be high, measuring the thickness of the pipeline wall may be useful and more expedient. But in the conditions of local carbon dioxide corrosion, to obtain a more accurate assessment, radiography testing is used together with ultrasonic testing. This is due to the fact that the place and time of the formation of a local defect is impossible to predict, which limits the possibility of using non-intrusive methods in the absence of data on the location of the defect [15].

\section{Optimization of corrosion monitoring and corrosion protection}

All methods of corrosion control are applied to obtain a complete assessment of the corrosion condition of an object. The extent to which a particular method is involved depends on the specific operating conditions and challenges faced by corrosion monitoring and corrosion protection services. Direct methods of corrosion monitoring, as discussed in the previous section, include thickness measurement and detection of thinning and defects in pipes and equipment. With their help, it is possible to record only the current corrosion state, but it is impossible reliably (with satisfactory accuracy) predict the development of corrosion processes in conditions of changes in the technological parameters of the object during operation [16]. Diagnostic methods have limitations. The configuration and parameters of local defects (pits and their clusters, figure 4) can create difficulties in the accuracy of results and data interpretation. Diagnostics is carried out periodically and selectively at the location, and also fixes already existing local defects: diagnostics follows the corrosion process with a delay due to the sensitivity of the method and the frequency of control. It is difficult to know in advance the place and time of the formation of defects, and it is impossible to inspect the entire surface of the pipelines.

To increase information support for the assessment of corrosion processes, taking into account the control of corrosion conditions and factors, it is rational to use 
diagnostic examinations taking into account the results of other methods of corrosion monitoring (direct and indirect), including simulation tests.
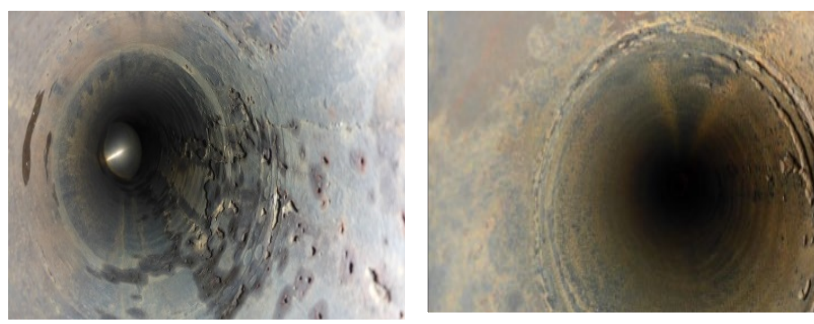

Fig. 4. Examples of typical localized corrosion damage due to $\mathrm{CO} 2$ corrosion [1].

Simulation tests make it possible to rank, according to the degree of corrosiveness, different areas where the operating conditions and, accordingly, some corrosion parameters change. Condensation moisture and elevated temperature are the main intensifiers of the corrosion process. Potential areas can be identified where favorable thermobaric conditions are created and moisture condensation occurs, taking into account the decrease in the temperature of the environment as the produced hydrocarbons move along the pipeline system.

Also, carrying out simulation tests allows predicting the dynamics of corrosiveness of environments, taking into account the possible change in corrosive factors over time. For example, a decrease in the total pressure, typical for all hydrocarbon fields, will lead to a decrease in the partial pressure of $\mathrm{CO}_{2}$ with the time of operation.

Our analysis shows that sections of the pipeline system of a gas pipeline can be ranked by assessing the degree of corrosiveness of the transported medium. Such a ranking is carried out by the type of corrosion (TOL and BOL), taking into account changes in corrosionhazardous factors (the type of water environment, the appearance of formation water, temperature, partial pressure of $\mathrm{CO}_{2}$, etc.). In the first sections of the pipeline, the largest temperature drop will occur, leading to localized corrosion damage TOL corrosion. In further sections, pipelines in contact with the $\mathrm{CO}_{2}$ medium will be subject to mainly BOL corrosion, when the separation and accumulation of corrosive aqueous medium along the bottom of the pipe will occur. Taking into account these and other factors and the ranking of the pipeline system, the selection of the optimal means of protection against corrosion and the organization of the corrosion monitoring system should be carried out. The choice of the corrosion inhibitor and the technology of its application is also carried out taking into account the operational factors and the type of development of TOL or BOL corrosion.

\section{Conclusions}

- The basis for the correct assessment and forecast of the development of the corrosion situation of gas production facilities is a set of studies, including the analysis of the initial operating conditions of the field, simulation corrosion and analytical test methods.
- When assessing a corrosion situation, the entire possible range of values of the main corrosion parameters should be considered, taking into account their expected changes during the life cycle of the production facility. The main types of corrosive attacks at gas production facilities are BOL and TOL corrosion.

- The applied corrosion inhibitors should satisfy a number of protecting and technological requirements. Efficiency control is carried out according to corrosion monitoring data.

\section{References}

1. D.N. Zapevalov, R.K. Vagapov, K.A. Ibatullin, Science \& Technology in the Gas Industry, 3 (75), 59 (2018)

2. L.L. Rozenfeld, L.V. Frolova, V.M. Brusnikina, OPA Ltd., Vol. 8, P. 115 (1987)

3. GOST R 58284-2018, Petroleum and natural gas industries. Offshore installations and pipelines. General requirements for corrosion protection

4. GOST R 55990-2014 Oil and gas-oil fields. Field pipelines

5. A.I. Fedotova, R.K. Vagapov, D.N. Zapevalov, K.O. Strelnikova, E3S Web Conf., 4, 59, (2018)

6. D.N. Zapevalov, R.K. Vagapov, E3S Web of Conferences 121, 02013, (2019).

7. R.K. Vagapov, D.N. Zapevalov, K.A. Ibatullin, Voprosy Materialovedeniya 1 (101), 163, (2020)

8. M. Singer, Corrosion 73, 1030 (2017)

9. Y.M. Gunaltun, D. Larrey, Oil \& gas journal, 28 (98), 58, (2000)

10. K.O. Strelnikova, R.K. Vagapov, D.N. Zapevalov, A.I. Fedotova. Transport and storage of oil products and hydrocarbons, 2, 16, (2018)

11. STO Gazprom 9.3-011-2011 Corrosion protection. Protection of field facilities against corrosion by using corrosion inhibitors. Basic requirements

12. B. Hedges, S. Papavinasam, T. Knox, K. Sprague, Corrosion, Paper 5503, (2015)

13. R.K. Vagapov, D.N. Zapevalov, Science \& Technology in the Gas Industry 2 (82), 60, (2020)

14. Y. Zhang, G. Yan, Defectoskopiya 2, 78, (2007)

15. D.N. Zapevalov, R.K. Vagapov, Testing. Diagnostics, 3 (261), 30, (2020)

16. R.K. Vagapov, D.N. Zapevalov, Defectoskopiya 7, 61, (2020) 